\title{
Geometry of Martian impact craters: First results from an interactive software package
}

\author{
Peter J. Mouginis-Mark, Harold Garbeil, and Joseph M. Boyce \\ Hawaii Institute of Geophysics and Planetology, University of Hawaii at Manoa, Honolulu, Hawaii, USA
}

Celine S. E. Ui

Sociology Department, University of Washington, Seattle, Washington, USA

Stephen M. Baloga

Proxemy Research, Laytonsville, Maryland, USA

Received 27 June 2003; revised 1 June 2004; accepted 21 June 2004; published 24 August 2004.

[1] We have developed a new interactive computer program that facilitates the easy collection of geomorphic data for Martian impact craters, using the MOLA 128th degree digital elevation model of Mars. We describe the method for ensuring that accurate measurements of crater diameter, depth, rim height, rim volume, cavity volume, ejecta thickness, and ejecta volume are obtained. We compare our measurements of crater diameters and rim heights to results obtained by Garvin et al. [2000], who employed centerline MOLA profiles. Statistical regressions between the two methods give $\mathrm{R}^{2}$ values of 0.930 for crater depths and 0.984 for crater diameters. The new interactive program facilitates the rapid compilation of large data sets to allow a comparison of crater populations in different settings. Preliminary results are presented for 354 craters on ridged plains materials in Hesperia and Sinai Plana to demonstrate the value of the program for regional comparisons and the analysis of degradational processes on Mars. INDEX TERMS: 5420 Planetology: Solid Surface Planets: Impact phenomena (includes cratering); 5494 Planetology: Solid Surface Planets: Instruments and techniques; KEYWORDS: geomorphology, impact craters, Mars

Citation: Mouginis-Mark, P. J., H. Garbeil, J. M. Boyce, C. S. E. Ui, and S. M. Baloga (2004), Geometry of Martian impact craters: First results from an interactive software package, J. Geophys. Res., 109, E08006, doi:10.1029/2003JE002147.

\section{Introduction}

[2] Since the earliest days of the Viking Orbiter missions, it has been recognized that fresh impact craters on Mars have interior and exterior features that often differ from those seen on the Moon and Mercury [Carr et al., 1977; Wood et al., 1978; Mouginis-Mark, 1979]. These differences can be seen in the morphology of the ejecta deposits, the depth/diameter relationships, and the size and frequency of central peaks [Barlow and Bradley, 1990]. The geometry of the crater cavity provides insights into the cratering process and the distribution of ejecta. Spatial variations in the depth/diameter ratio for craters of comparable size might indicate variations in target properties [Cintala et al., 1976; Pike, 1980a], degradation processes [Soderblom et al., 1974] or the distribution of subsurface volatiles [Cintala and Mouginis-Mark, 1980]. Quantitative analysis of the thickness of ejecta layers around the parent crater may also provide information on the rheology of the ejecta at the time of emplacement, thereby enabling differences in the volatile distribution of the target [Gault and Greeley, 1978; Baratoux et al., 2002] or the effects of the atmosphere [Barnouin-Jha and Schultz, 1998] to be investigated.

Copyright 2004 by the American Geophysical Union. 0148-0227/04/2003JE002147\$09.00
[3] Until recently, however, the development of large data-bases for Mars craters has required considerable amounts of time either to carefully measure photographic prints [e.g., Pike, 1980a] or inspect individual altimetric profiles obtained by the Mars Orbiter Laser Altimeter (MOLA) [Garvin and Frawley, 1998; Garvin et al., 2000]. Garvin et al. [2000] also employed a semiautomated measurement system for quantifying a broad suite of geometric data for craters. To further this collection of geometric data, we have developed an interactive computer program to investigate the geometry of Martian impact craters. The program, called IMPACT ("Interactive Measurement, Profiling, and Analysis of Crater Topography") is written in C++ for MS Windows-based personal computers, so that it will run on any moderately powerful PC running Windows 98/NT/XP/2000. This program will be made available to the community via the Mars Crater Consortium [Barlow et al., 2000] and the U.S. Geological Survey, Flagstaff, Arizona.

[4] Our long-term objective is to search for geologically significant global spatial variations in crater geometry (e.g., depth/diameter, rim volume/rim height, or ejecta volume/ crater volume) that might indicate variations in target properties or the resurfacing history of Mars. Here we describe the techniques and computational algorithms incorporated within IMPACT to produce the geometric 

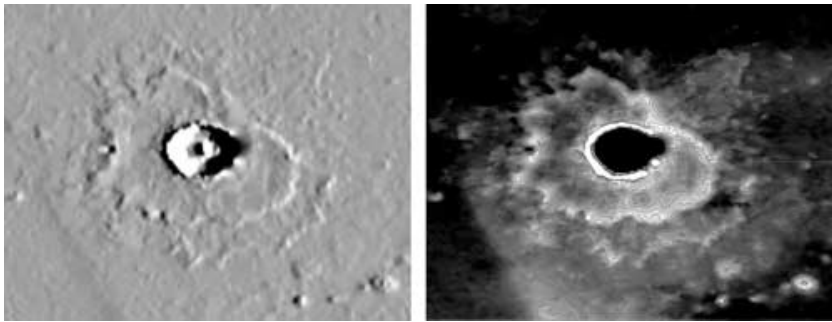

Figure 1. Two versions of the MOLA topography for the crater Arandas, which is located at $42^{\circ} \mathrm{N}, 345^{\circ} \mathrm{E}$. At left is a shaded relief version derived from the 128th degree MOLA DEM, with the lighting coming from the right and an inclination of 30 degrees. At right is a stretched elevation image with low as black and high as white. Contours at $50 \mathrm{~m}$ intervals are superimposed on the image. In both of these renditions, a low point can be identified on the right-hand rim of the crater. Inspection of the map showing the distribution of MOLA profiles across this crater (Figure 2) illustrates that this is a part of the crater where no MOLA data were collected, and so there is the potential for measurement errors for this part of the crater.

variables for an impact crater. We also show how our results compare to those of Garvin et al. [2000], who investigated individual center-line MOLA profiles to determine some of the same parameters. Finally, we present new geometric data for craters on the ridged plains of Mars as a demonstration of the value of the IMPACT program in comparing different crater populations.

\section{Limits of Program}

[5] We use the 128th degree MOLA digital elevation model as our input data. Investigators working with IMPACT must be aware of both the strengths and the limitations of the program for conducting regional or global studies of impact craters on Mars. As has been discussed by Garvin and Frawley [1998] and Garvin et al. [2000], the limiting factor in working with MOLA data for the determination of impact crater geometry is the spatial distribution of the laser point measurements on the surface. MOLA data have a non-uniform distribution both in latitude and longitude. Potentially, no MOLA measurement lies on the floor of the smaller craters, so that the derived value of crater depth will in reality come from the inner crater wall, giving a depth that is too shallow. Similarly, the crest of the crater rim may also be missed due to the along-track spacing of the MOLA point measurements. In this instance, the measured rim height would be too small.

[6] It is not possible for us to give a minimum size for craters that can be measured with IMPACT because of the wide variety of geometric parameters that can be measured, but we caution against over interpreting IMPACT results for smaller craters and landforms. Because of the convergence of orbits closer to the poles, MOLA coverage is better at high latitudes and poorer close to the Equator. For a given size of landform, it is likely that IMPACT will give a more representative measurement at higher latitudes because of the greater number of MOLA measurements per unit area. In addition, the physical size of the landform is important because of the $160 \mathrm{~m}$ diameter footprint, and 300 meter along-track spacing, of the MOLA measurements [Zuber et $a l ., 1992]$. It is more likely that individual MOLA shots hit the floor of a crater compared to, for instance, the crest of an ejecta rampart due to the different relative sizes of these two types of landform. Maximum rim heights would also tend to be under estimated. In instances where data on crater depth and rim height need to be collected for small craters, we recommend the visual selection approach taken by Garvin and Frawley [1998] and Garvin et al. [2000]. In contrast, crater diameter, rim volume, and the planimetric area of ejecta layers, can be confidently measured.

[7] There are many issues in data quality to be aware of when making geometric measurements of craters using the MOLA data set [cf. Garvin and Frawley, 1998; Garvin et al., 2000]. In Figure 1 we show two renditions of the $28 \mathrm{~km}$ diameter crater Arandas. In both the shaded relief image and the stretched elevation data, it is evident that something is odd about the eastern rim. Inspection of the distribution of MOLA profiles across the crater (Figure 2) shows that this feature is due to the lack of elevation data for the eastern rim. To avoid this problem in data sampling, IMPACT allows the interactive viewing of the MOLA 128th degree "hit count" map, which shows how many data points lie within a pixel of the DEM. It is then possible to select points on the rim that contain real elevation data, rather than interpolated values.

[8] Problems may also arise when measuring the crater planimetric shape. Our program allows one to digitize the rim crest, the perimeter of the outer crater wall, and the perimeter of the ejecta lobes (Figure 3). As many points as necessary can be collected for each attribute. At each step, the user can switch between different versions of the topographic data to get the best lighting geometry to see the feature being mapped, as shown in Figure 4. Shaded relief images with any combination of solar elevation and azimuth can be used as this base image. Once each attribute of the crater has been defined, the program automatically displays the part of the crater that will be included in the height and volume calculations. If any part of the ejecta blanket lies below the defined surface, then the pixels are not illuminated. This provides the user with another visual check on the quality of the derived values.

[9] The IMPACT program may not therefore be the optimum tool for the detailed analysis of crater topography in the same way that Pike [1980b] investigated the geometry of lunar craters. IMPACT does not allow one to define the floor diameter for a crater, nor do we provide the capability to exclude the topography of a central peak to be removed from the calculation of crater depth. This lack of capability is primarily due to the limited spatial resolution of the MOLA DEM (450 $\mathrm{m}$ at the equator). However, as higher spatial resolution topographic maps for Mars are produced from the Mars Express stereo camera [Neukum and the HRSC Co-Investigator Team, 2004] or future missions, it will be straightforward to modify IMPACT to determine additional crater attributes.

\section{Defining the Preimpact Reference Surface}

[10] The most important step in data collection is to define the "preimpact reference surface" or the inferred 


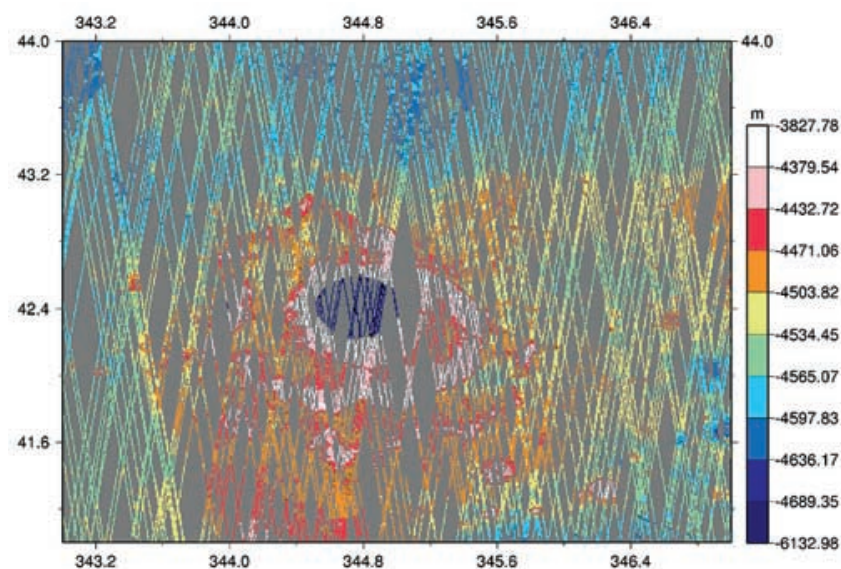

Figure 2. Map showing the distribution of MOLA profiles across Arandas crater. Colors represent elevations, which are keyed to the elevation bar at right. Notice the lack of profiles across the eastern rim, which has produced the errors in the interpolated data shown in Figure 1.

topography of the landscape prior to the formation of the crater. It is against this reference surface that all subsequent parameters (e.g., height, depth, and volume) are measured. If this surface is incorrect (either warped relative to the real topography, or with a vertical offset in elevation), then none of our derived geometric values will be accurate.

[11] The preimpact reference surface is obtained for each crater by selecting at least eight (and typically 20-25) points beyond the range of the ejecta. IMPACT employs a general least squares routine [Press et al., 1992] to fit a general second-order polynomial to the elevations identified by the investigator. We have found that the best results are obtained when the points are scattered over a wide range of azimuths and radial distances away from the crater (ideally out to $\sim 10$ crater radii from the rim crest). Care must be taken when studying craters located on hilly terrain so that low points on the surrounding terrain are selected; otherwise, an inappropriately high flat surface equivalent to the tops of the hills would be computed.

Figure 3. The IMPACT program provides visual checks of the quality of the collected data at different stages of the measurement process, which can then be corrected if errors are detected. In the top panel we show the calculated surface area of the crater as defined by digitizing the rim crest. If there were bad data in this measurement, parts of the crater interior would not show up in color. In the middle, we show the extent of the outer rim, which defines the volume of the rim crest. Again, if there were errors in this measurement, the problem areas would not be colored. At bottom we show the inferred distribution of the ejecta layer. In this instance, parts of the surface of the ejecta blanket (south of the crater) lie below the inferred level of the preimpact surface. Those parts of the ejecta blanket that are not subsequently included in the calculation of ejecta volume are shown by the lack of color. Crosses mark the locations of points selected to help define the flat surface. This crater is $28 \mathrm{~km}$ in diameter and is located just west of Olympus Mons aureole (see also Figure 4).
[12] A second-order polynomial is generated as a model of the preimpact topography. This allows for the possibility that the surface was not originally flat. This polynomial has the form

$$
E=a_{0}+a_{1} * X+a_{2} * Y+a_{3} * X^{*} Y+a_{4} * X^{2}+a_{5} * Y^{2},
$$

where $\mathrm{E}$ is the preexisting surface elevation, $\mathrm{X}$ is the sample location, $\mathrm{Y}$ is the line location, and $\mathrm{a}_{0}, \mathrm{a}_{1} \ldots \mathrm{a}_{5}$ are the coefficients estimated from nearby elevations by the least squares fitting routine. These $\mathrm{X}$ and $\mathrm{Y}$ locations are the same as the latitude and longitude in the DEM.

[13] Besides a simple plane, shapes like elliptic bowls and domes, as well as warped surfaces with both convex upward and downward curvatures, can be obtained as reference
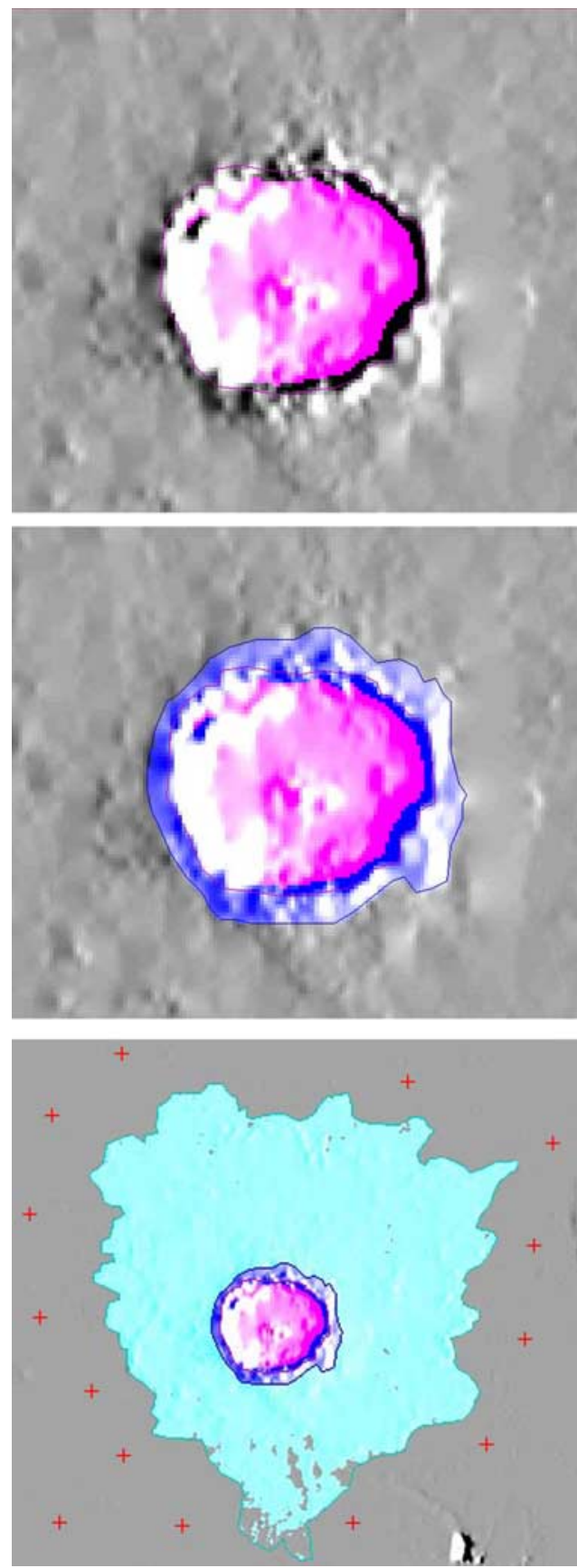

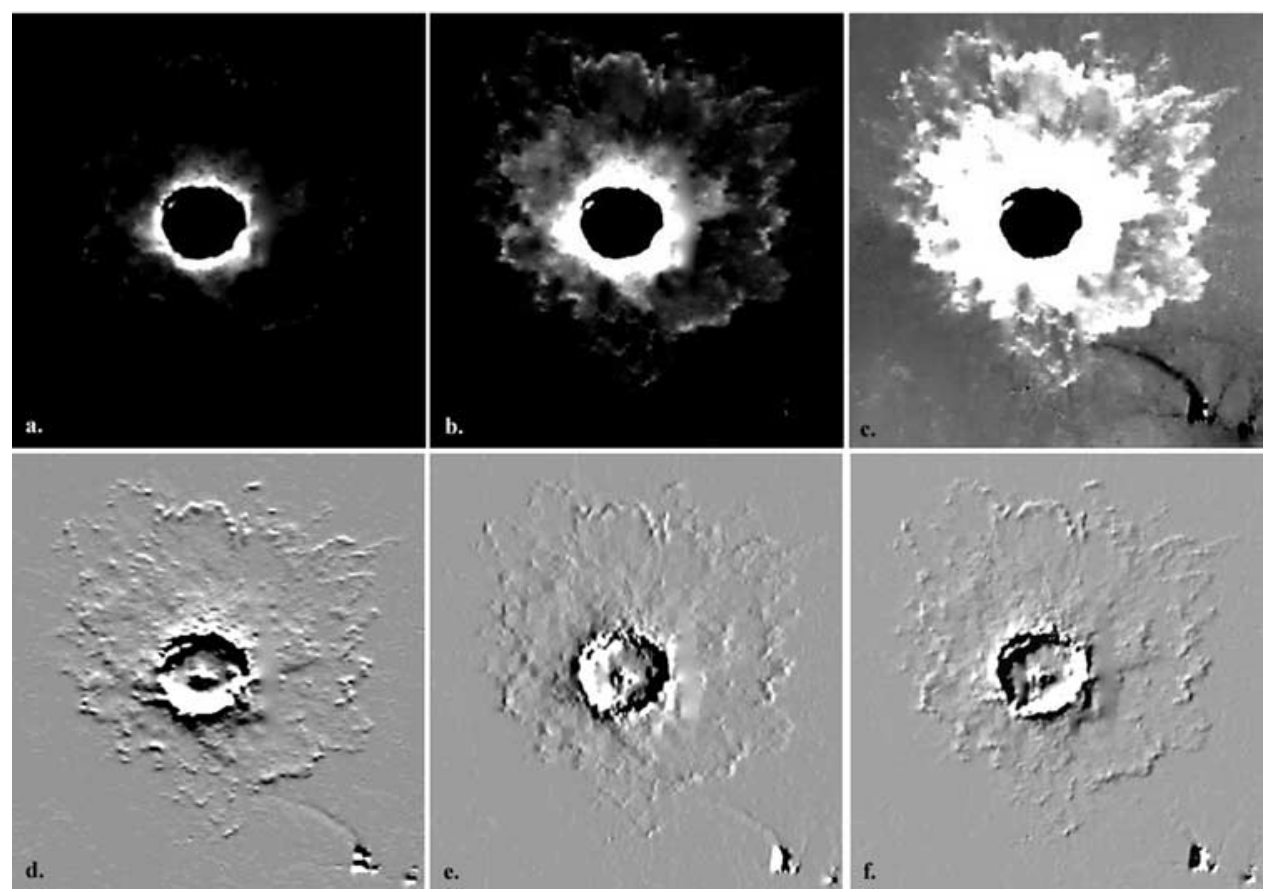

Figure 4. Six different versions of the same MOLA data for a $28 \mathrm{~km}$ diameter crater just west of the Olympus Mons aureole. Any scenes like these can be shown using IMPACT to help define the rim crest, exterior rim wall, the distal edges of the ejecta layers, or other morphologic attributes. The geometry of this crater is given in Table 1. (a) Elevation data stretched over the range -3811 to $-3487 \mathrm{~m}$ relative to the MOLA datum; (b) elevation data stretched over the range -3866 to $-3700 \mathrm{~m}$; (c) elevation data stretched over the range -3894 to $-3838 \mathrm{~m}$; (d) shaded relief image, with the illumination azimuth of $10^{\circ}$ and a solar elevation of $25^{\circ}$; (e) shaded relief image, with the illumination azimuth of $90^{\circ}$ and a solar elevation of $25^{\circ}$; and (f) shaded relief image, with the illumination azimuth of $300^{\circ}$ and a solar elevation of $25^{\circ}$

surfaces. The values of the parameters in the fitted polynomial, and estimates of their statistical confidence limits, are available in the output for the inspection. The quality of the overall fit of the preimpact surface and the values of the each of the individual parameters can therefore be evaluated [Selby, 1973; Draper and Smith, 1981; Sheskin, 1997] to determine which type of reference surface provides the best model.

[14] IMPACT allows visual inspection of the surface-fit prior to data collection using multiple orientations of profiles running through the center of the crater (Figure 5). For example, if the derived surface results in the ejecta being below the surface then additional control points beyond the ejecta blanket can be selected. Areas with pronounced regional slopes, such as the flanks of the Tharsis and Elysium volcanoes, have also caused problems because the polynomial curve-fitting gives a surface that is not representative of the preimpact surface over distances in excess of $\sim 100 \mathrm{~km}$. This issue of curve-fitting to the landscape around the crater on the flanks of volcanoes is an inherent problem when using IMPACT since these landforms are not flat, nor do they have uniform curvature. Inspection of each best fit curve as it is calculated from different tie points while using the graphic interface (Figure 5), and then selecting profiles that fit below the crater profile is the best way to avoid this problem of IMPACT when measuring craters in topographically rough (at the tens of kilometer horizontal scale) parts of Mars. To a lesser extent, this problem may also be encountered in heavily cratered terrain if some of the reference points are selected on the floors of large craters and some points are placed on the tops of hills or the rims of these same craters.

\section{Measured Parameters}

[15] Figure 6 shows the parameters that can be measured for each crater using the IMPACT program. We emphasize that the primary application of these measured parameters is to rapidly generate large databases pertaining to crater geometry that can assist in the identification of regional trends [e.g., Boyce et al., 2003, 2004]. If the objective is to obtain the best quality geometric data for a few craters, then we recommend using the raw MOLA profiles and manual inspection of the elevation data overlying image data with at least $100 \mathrm{~m} /$ pixel spatial resolution.

[16] IMPACT is primarily intended for the analysis of Martian craters that have either a single ejecta layer or double ejecta layer, as defined by Barlow et al. [2000]. The outermost ejecta layer of a multiple ejecta layer crater (the MLE craters of Barlow et al. [2000]) could also be measured within IMPACT, although some MLE deposits are not complete around the crater, thus making it difficult to quantify them in this manner. Due to the resolution limit of the MOLA DEM, we have not found it possible to clearly 


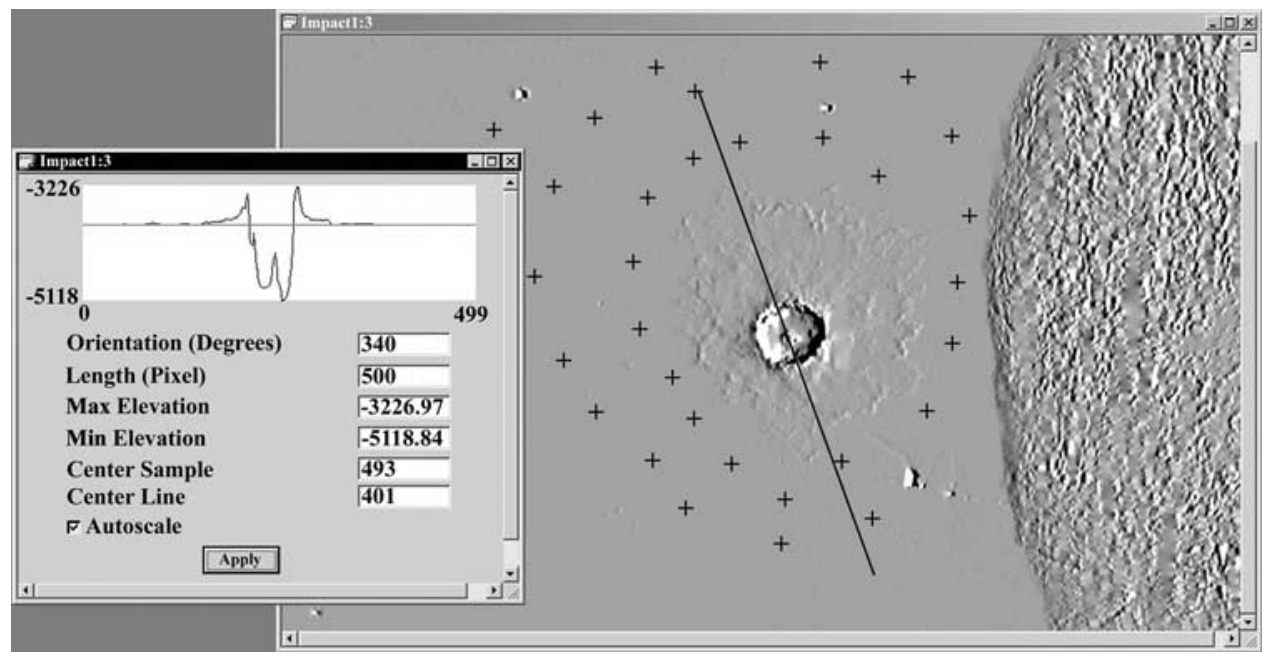

Figure 5. Example of the display screen presented by IMPACT to facilitate the selection of a flat surface surrounding the crater. The example shown is the same crater that is illustrated in Figure 4. The base image is a shaded relief image derived from MOLA topography. The black crosses are the selected points for the surface calculation. The line represents the test profile through the crater center that is also displayed in the window at left. Here the estimated flat surface is projected on top of the profile through the crater. If there is appreciable deviation between the fit and the real topography, the investigator must return to the program to select additional points to define the surface for a range of distances from the crater being studied.

define the boundary of multiple ejecta layers without using additional image data (say, from the Thermal Emission Imaging System (THEMIS) on the Mars Odyssey spacecraft). The current version of IMPACT does not provide online access either to THEMIS or Viking images to help define the type of ejecta surrounding a crater, but future enhancements to IMPACT will include the ability to view THEMIS daytime IR images (at $100 \mathrm{~m} /$ pixel resolution) as a supplemental data set.

[17] The IMPACT program generates three files for each crater. These files include one providing the surface polynomial coefficients and goodness of fit statistics, a file with vertices of the defined features, and a descriptive results file. The program also appends the results to an ASCII file which is readily importable into a spreadsheet or plotting program. This file writes a single line of information for each file with each line consisting of 19 columns, summarized as follows:

[18] 1. Crater name: Assigned at the start of each set of measurements.

[19] 2. Crater latitude: The geographic latitude of the crater is selected while viewing the outline of the crater. The location of all vertices is also stored in a coordinate file for importation into a geographic information system (GIS) if desired.

[20] 3. Crater longitude: The geographic longitude of the crater is selected while viewing the outline of the crater.

[21] 4. Crater elevation: The elevation is determined for the same location as parameter 2 . It is defined as the elevation of the crater with respect to the best fit surface. The elevation is expressed in meters above the MOLAdefined datum.

[22] 5. Equivalent crater diameter: For craters larger than $\sim 5 \mathrm{~km}$ in diameter, as many as $10-25$ points around the rim are measured to define the rim crest. These points define a polygon, with the rim crest being the points lying on the polygon. IMPACT also stores all of the latitude/longitude information for the selected points on the rim to facilitate ingestion of the data into a GIS database. The equivalent crater diameter is calculated as the diameter of a circle that has the same area as the area contained within the rim crest.

[23] 6. Maximum crater depth: Measured as the elevation difference between the average rim crest elevation and the maximum depth of the crater floor. It employs data from the rim crest measurements. This parameter is most helpful for studying simple bowl-shaped craters as it records the maximum depth of the crater. This attribute may also be useful in instances where, for example, a large central peak exists within a complex crater. A central peak would reduce the average crater depth obtained by IMPACT.

[24] 7. Mean crater depth: This is the parameter that is most often reported in the literature for Martian craters because it is the easiest to measure from shadow lengths [Pike, 1980a; Cintala and Mouginis-Mark, 1980]. Here we calculate the mean crater depth by subtracting the average elevation of all pixels within the rim crest from the average rim elevation (parameter 9). We choose this combination because the mean rim elevation is more likely to be



Figure 6. Cross section of idealized crater, showing parameters measured. 
representative of the rim topography than the maximum elevation, and because it is easy to measure with IMPACT. We note that for some craters with shallow inner walls, there may be several pixel values from the inner wall that are included in this average. This is also true if there is a central peak on the crater floor. Because of the spatial resolution of the MOLA DEM, we have not attempted to separate these three attributes (i.e., crater inner wall, flat floor, and central peak) because they are often very close (a few pixels) together.

[25] 8. Cavity volume: Calculated by taking each pixel within the DEM that lies below the preimpact surface and within the crater rim crest. Within IMPACT, these pixels are extended upward until they intersect the preimpact surface. The dimensions of these pixels, in meters, are calculated from the latitude of the point in the 128th degree DEM. These dimensions allow a pixel volume to be calculated. All of these individual pixel volumes are then summed to give the cavity volume.

[26] 9. Rim height: This is the mean elevation of the profile drawn around the rim crest (data from parameter 5 above) compared to the elevation of the preimpact surface. It is the mean height of a single line running around the crest of the rim.

[27] 10. Rim volume: Calculated by first delineating at least 3 points that mark the boundary between the rim and the inner ejecta lobe. As with the identification of the crater rim, the number of points around the exterior rim boundary typically varies from $\sim 10-25$ points depending upon crater size. This boundary can be mapped from multiple versions of a shaded relief image to select the best lighting geometry (i.e., solar elevation and azimuth) for each part of the rim (see section 5). The rim volume is derived using all the points within this perimeter and the place where the inner crater wall intersects the preimpact surface.

[28] 11. Number of ejecta points below reference surface: Even for craters that formed on a smooth target, we have found that our best fit surface sometimes lies above points on the ejecta blanket. This may be due to real undulations in the ejecta topography (due, say, to the presence of secondary craters) or to the curve-fitting of our reference surface. The reported number of points within the ejecta blanket that lie below the surface provides a measure of confidence for the derived average ejecta thickness and volume. It is expressed as a percentage of all points within the perimeter of the ejecta lobes.

[29] 12. Inner ejecta lobe volume: The investigator can digitize as many points as necessary to mark the edge of the inner ejecta lobe. The volume of material within the inner lobe that lies above the preimpact surface is calculated from this perimeter and the edge of the rim. All of the latitude/ longitude values selected are automatically stored for possible data inclusion in a GIS layer.

[30] 13. Inner ejecta lobe mean thickness: The same boundaries as defined for parameter 12 are employed here. Calculated as the mean height between the preimpact surface and the surface of the inner ejecta lobe. Any points within the inner lobe below the preimpact surface are flagged and discounted.

[31] 14. Inner ejecta lobe area: This is the planimetric area of the ejecta lobe and includes all the pixels within the boundaries as defined for parameter 12. IMPACT converts
Table 1. Example of IMPACT Output for a Crater in Amazonis Planitia $^{\mathrm{a}}$

\begin{tabular}{|c|c|}
\hline & Name/Value \\
\hline \multicolumn{2}{|c|}{ Location Information } \\
\hline Crater name & Amazonis1 \\
\hline Latitude & 23.1953 \\
\hline Longitude & 207.758 \\
\hline Pixel dimension & $442.4 \mathrm{~m}$ \\
\hline
\end{tabular}

\begin{tabular}{ll}
\multicolumn{2}{c}{ Flat Surface Fitting Statistics } \\
Number of tie points & 35 \\
Tie pts. residual std. deviation & 2.5 \\
Tie points $\mathrm{R}^{2}$ & 0.71
\end{tabular}

Diameter

Crater Dimensions

Maximum depth

Average depth

Median depth

Total area of crater cavity

Cavity volume

$28.3 \mathrm{~km}$

$1261.3 \mathrm{~m}$

$534.5 \mathrm{~m}$

$736.3 \mathrm{~m}$

$629.7 \mathrm{~km}^{2}$

$379.0 \mathrm{~km}^{3}$

Maximum height

Rim Profile Statistics

Average height

Median height

Avg. height to crater max depth

$866.6 \mathrm{~m}$

$481.3 \mathrm{~m}$

$444.0 \mathrm{~m}$

$1742.6 \mathrm{~m}$

Area

Rim Parameters

Volume

$447.6 \mathrm{~km}^{2}$ $154.9 \mathrm{~km}^{3}$

Maximum thickness

Inner Lobe Statistics

Average thickness

Median thickness

Area

Volume

${ }^{\mathrm{a}}$ Mouginis-Mark et al. [2003].
$443.2 \mathrm{~m}$

$36.4 \mathrm{~m}$

$22.0 \mathrm{~m}$

$9287.0 \mathrm{~km}^{2}$ $309.6 \mathrm{~km}^{3}$ the number of pixels within the inner ejecta lobe to a surface area.

[32] 15. Outer ejecta lobe volume: This is determined in the same manner as parameter 12, except that the perimeter of the outer lobe and the perimeter of the inner lobe as the extent of the deposit are utilized.

[33] 16. Outer ejecta lobe mean thickness: Determined in the same manner as parameter 13, except that the perimeter of the outer lobe and the perimeter of the inner lobe as the extent of the deposit are utilized.

[34] 17. Outer ejecta lobe area: Determined in the same manner as parameter 14, except that the perimeter of the outer lobe and the perimeter of the inner lobe as the extent of the deposit are utilized.

[35] 18. Floor fill, mean thickness: Calculated for all craters, but is relevant only where the floor is above the level of the preimpact terrain. This is computed as the difference between the mean floor elevation (which is defined as the mean of all points within the rim crest) and that of the surrounding surface.

[36] 19. Floor fill minimum thickness: This is the minimum thickness of the points measured in parameter 18 .

\section{First Results}

[37] Table 1 presents the data output from our program for a $28 \mathrm{~km}$ diameter crater in Amazonis Planitia [MouginisMark et al., 2003], which is the same crater shown in 

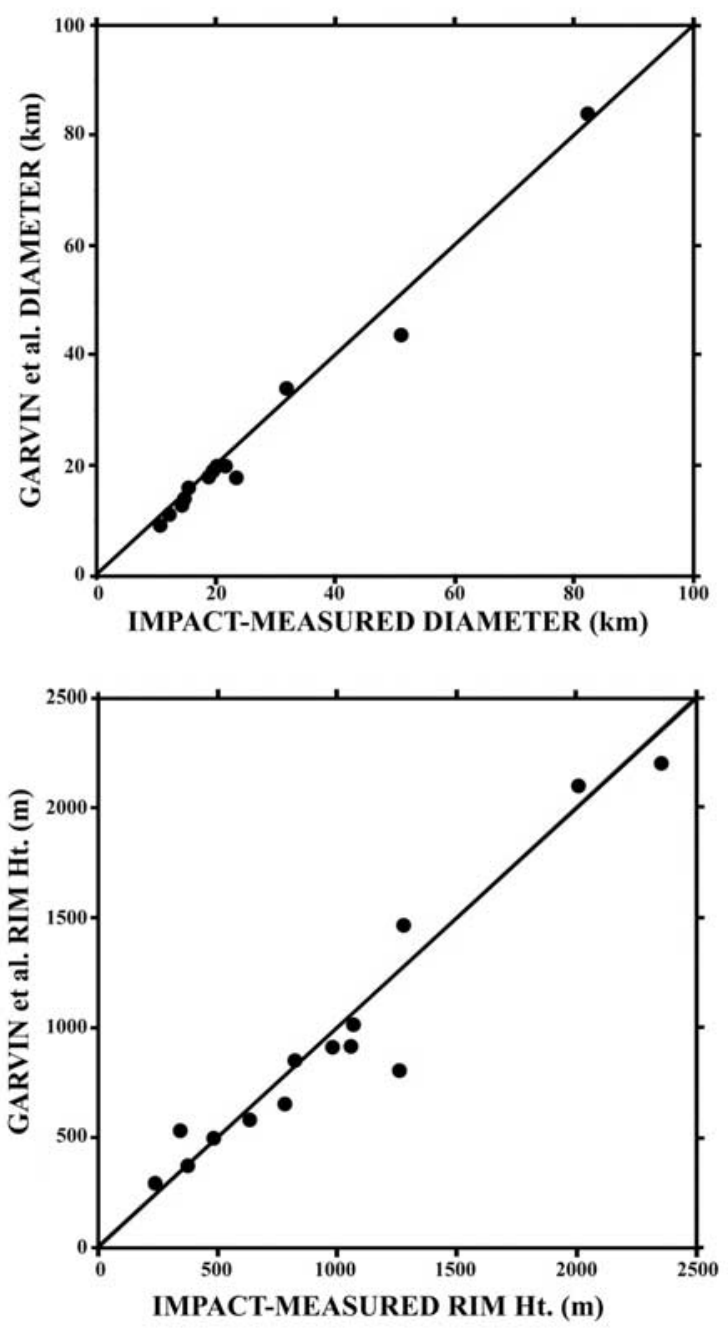

Figure 7. Comparisons of measured parameters obtained by the IMPACT program and the data for the same craters from Garvin et al. [2000]. At top we show a comparison of crater diameter, and at bottom we show crater depth.

Figures 4 and 5. Notice that several different parameters are listed along with the geometry of the crater in order to assess the quality of the measurements. In addition, in the program output, we also provide a trace along the rim crest of the crater. Often we have found that there is a significant amount of variability in the rim itself, so that the operator has to select which value of rim height is most meaningful to compare with other craters. For this example crater, it has an average height of $481 \mathrm{~m}$ but a maximum of $866 \mathrm{~m}$. This topographic variability of the rim has implications for earlier studies of Martian craters and other geologic processes, including the assumption that low crater rim heights arise from partial flooding of the crater exterior by lava flows [De Hon, 1982]. It was assumed that fresh impact craters on Mars have a constant depth to rim height ratio. De Hon [1982] developed this technique to estimate the thickness of lava flow sequences in the Eastern Tharsis region, and Greeley [1987] extended the approach to estimate the amount of juvenile volatiles that might have been released by volcanic plains units over geologic time. In both instances, had the factor of two in azimuthal variability of rim height been recognized, the thickness of lava flows and hence amount of juvenile volatiles released would have been much less constrained.

[38] A rigorous test of the IMPACT program is to compare our measurements with the results of Garvin et al. [2000], who carefully selected dimensional data from individual MOLA profiles that run through the center of the craters. The graphs in Figure 7 show the results of this comparison for the polar craters listed in Table 2 of Garvin et al. [2000]. These craters have a diameter range of 9$84 \mathrm{~km}$, a depth range of $0.30-2.2 \mathrm{~km}$, and a distribution in latitude from $72^{\circ}-85^{\circ} \mathrm{N}$.

[39] While some differences exist between the two data sets, the $\mathrm{R}^{2}$ values are 0.930 for the crater depths and 0.984 for crater diameters. These correlations are extremely good considering the differences in the methods of analysis. There are several explanations as to why some of the results derived from IMPACT differ from those obtained from the individual MOLA profiles, albeit the best profile based on visual inspection to determine which profile crosses the center of the crater. In terms of measuring crater diameter, IMPACT provides the equivalent crater diameter based on the area contained within the rim crest. In the case of the Garvin et al. [2000] data set, only one measure of the diameter could be made along the MOLA profile. This approach would produce an identical diameter if the crater were perfectly circular, for a polygonal crater rim it is possible that a single profile might either cross an unusually wide or narrow part of the crater leading to a discrepancy between the results of the two methods.

[40] A second issue concerns the ability of the interpolated MOLA DEM to accurately define the crest of the rim. Single MOLA measurements that represent individual high points on the rim will always be resampled to the 128th degree spatial resolution of the DEM, and the absolute elevation will be lower than the true value. However, we do not consider this averaging to be a major problem in our database at the few percent level, since all reported measurements will have the same bias. Trends in the database from one region to another will therefore be valid and such biases can be accounted for.

[41] We have compiled geometric data using IMPACT for relatively fresh impact craters located on several examples of ridged plains materials in Hesperia and Sinai Plana. These units have previously been advocated as good test areas for studying the effects of latitude and elevation on the morphology of impact craters [Horner and Greeley, 1987; Mouginis-Mark and Hayashi, 1993]. In this study, we explore possible differences in the general geometry of craters in these two areas. Table 2 gives our database, which includes 354 craters in the diameter range $2.4-89.3 \mathrm{~km}$. Figure 8 presents the depth/diameter data, and Figure 9 presents rim volume/diameter and rim volume/cavity volume measurements.

[42] Comparing our depth/diameter data to those collected by Pike and Davis [1984] and Garvin et al.

Table 2. Sample Data for Craters on Ridged Plains Materials

\begin{tabular}{|c|c|c|c|c|}
\hline Location & $\begin{array}{c}\text { Latitude } \\
\text { Range, }{ }^{\circ} \mathrm{S}\end{array}$ & $\begin{array}{l}\text { Longitude } \\
\text { Range, }{ }^{\circ} \mathrm{E}\end{array}$ & $\begin{array}{c}\text { Diameter } \\
\text { Range, km }\end{array}$ & $\begin{array}{l}\text { Number } \\
\text { of Craters }\end{array}$ \\
\hline Sina & $10.28-29.66$ & $261.53-278.56$ & 1.6 & 206 \\
\hline Hesperia Planum & $16.98-29.61$ & $107.1-118.5$ & $2.8-89.3$ & 146 \\
\hline
\end{tabular}




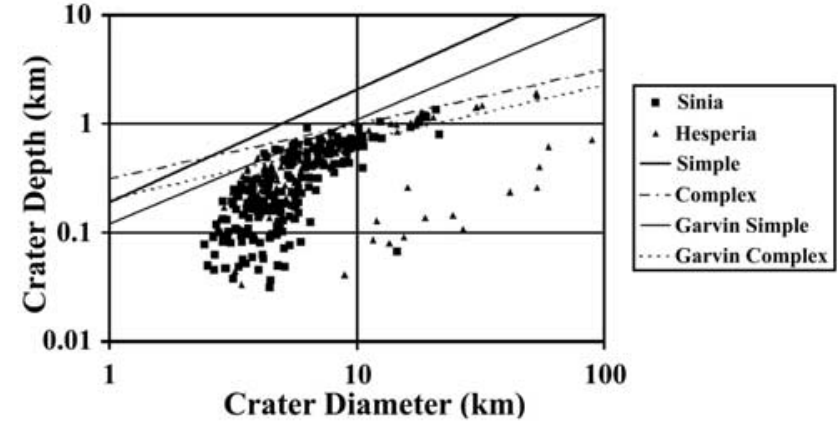

Figure 8. Preliminary depth/diameter data obtained using the IMPACT program for three areas of ridged plains on Mars. Craters were measured for Sinai and Hesperia Plana. Also shown are the best fit curves for simple and complex craters from Pike and Davis [1984] and Garvin et al. [2000]. The best fit curves labeled "Simple" and "Complex" are from Pike and Davis [1984]. Table 2 provides the latitude, longitude, and diameter range for these craters studied here.

[2000], Figure 8 demonstrates that using IMPACT produces a very similar relationship for large ( $>6 \mathrm{~km}$ diameter) craters (Figure 8). Indeed, our measurements of the deepest craters of a given size are bounded by the two least squares fits to the data from these earlier studies. Also apparent is the significant number of craters that are unusually shallow for their diameter, with 14 craters $\sim 9 \mathrm{~km}$ or larger in Hesperia Planum being shallower by $\sim 1 \mathrm{~km}$ than would be expected from their diameter. We have described unusually shallow craters elsewhere [Boyce et al., 2004] and believe that this reflects partial in-filling of the craters, but further geometric and morphologic analysis is needed to identify the process(es) responsible for producing these unusually shallow craters. Because IMPACT restricts our measurements to craters of several kilometers diameter, we do not believe that we have adequately sampled crater depths for this size range, and so do not draw any conclusions about the apparent depth/diameter relationship for craters $<6 \mathrm{~km}$ diameter that we have measured.

[43] Figure 9 presents crater data that are of types that have not previously been collected for Mars; only Pike [1980b] has collected comparable data for lunar craters. Rim volume is seen to vary by a factor of up to $\sim 50$ for craters of almost the same diameter over the diameter range 15-22 km, with no clear differences between the two geographic areas. Similarly, no regional differences appear in the comparison of crater cavity volume vs. rim volume for the two crater populations. Cavity volume is found to be $\sim 2-2.5$ times the rim volume, suggesting that a significant amount of material that was excavated from the crater is now distributed beyond the crater rim, or has been eroded from the crater. Quantitative results from a detailed analysis of these trends will appear elsewhere.

\section{Summary}

[44] The IMPACT program provides a relatively quick and easy way to interactively assemble a large database on the geometry of impact craters on Mars. We nevertheless caution against working with IMPACT without careful inspection of the distribution of the MOLA data points with respect to the landforms being measured. IMPACT contains a number of diagnostics to assist in obtaining the best estimates of crater feature dimensions. This feature provides a means for minimizing systematic errors in regional comparisons and enhances the ability to make more rigorously defensible scientific inferences.

[45] Our preliminary results compare favorably with the more time intensive effort of identifying and using individual MOLA profiles, and provide volumetric measurements that are not obtained from single profiles. Future studies of Martian craters are planned in order to explore the variation of ejecta, rim, and cavity volume as a function of latitude and target material, thereby providing numerical models of the cratering process [Stewart et al., 2001] with quantitative parametric information. Our preliminary inspection of crater geometric data for the Northern Plains [Boyce et al., 2003, 2004] also suggests that by using IMPACT a considerable amount of information may be derived about the erosional and depositional history of the area based on the depth/ diameter relationship of craters in Utopia Planitia. Further work to explore these opportunities is currently underway.

[46] IMPACT has been written to run on computers that are accessible to many planetary scientists. Investigators
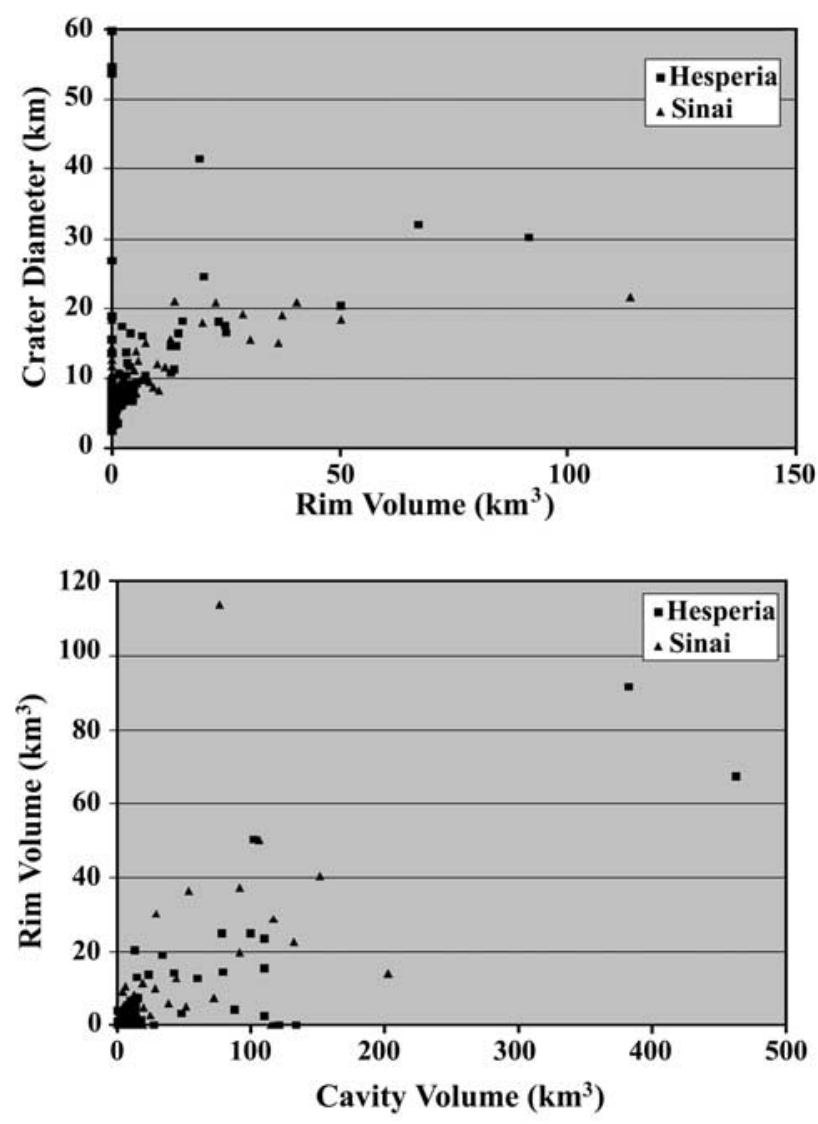

Figure 9. Preliminary volume measurements for the same craters in Sinai and Hesperia Plana that are presented in Figure 8. (top) Rim volume compared to crater diameter. (bottom) Cavity volume compared to rim volume. In this plot it is apparent that $<40 \%$ of the material that once occupied the cavity now resides within the rim of the crater. 
wishing to obtain a copy of IMPACT for their own investigations are encouraged to obtain a copy of the software from the US Geological Survey, Flagstaff, AZ, via the Mars Crater Consortium. A beta-version of the program, along with help information, is already available at http:// www.higp.hawaii.edu/ harold/Impact_Program/.

[47] Acknowledgments. We thank Jim Garvin for discussions on using the MOLA data set for making measurements of Martian impact craters. Scott Anderson is thanked for his preparation of Figure 2. Nadine Barlow, Richard Pike, and an anonymous person provided constructive reviews of an earlier version of this manuscript. This is HIGP paper 1336 and SOEST contribution 6398

\section{References}

Baratoux, D., C. Delacourt, and P. Allemand (2002), An instability mechanism in the formation of the Martian lobate craters and the implications for the rheology of ejecta, Geophys. Res. Lett., 29(8), 1210, doi:10.1029/ 2001GL013779.

Barlow, N. G., and T. L. Bradley (1990), Martian impact craters: Correlations of ejecta and interior morphologies with diameter latitude, and terrain, Icarus, 87, 156-179.

Barlow, N. G., J. M. Boyce, F. M. Costard, R. A. Craddock, J. B. Garvin S. E. H. Sakimoto, R. O. Kuzmin, D. J. Roddy, and L. A. Soderblom (2000), Standardizing the nomenclature of Martian impact crater morphologies, J. Geophys. Res., 15, 26,733-26,738.

Barnouin-Jha, O. S., and P. H. Schultz (1998), Lobateness of impact ejecta deposits from atmospheric interactions, J. Geophys. Res., 103, 25,73925,756

Boyce, J. M., P. J. Mouginis-Mark, J. B. Garvin, and H. Garbeil (2003), Evidence for a thick mantle of volatile-rich materials in the Utopia Basin, Mars, based on crater depth/diameter measurements, Lunar Planet. Sci. XXXIV, abstract 1967 .

Boyce, J. M., P. J. Mouginis-Mark, and H. Garbeil (2004), Depth to diameter relationships of craters in the high latitudes $(70-80)$ of Mars: Implications for geologic history of those areas, Lunar Planet. Sci., $X X X V$, abstract 1129 .

Carr, M. H., L. Crumpler, J. Cutts, R. Greeley, J. Guest, and H. Masursky (1977), Martian impact craters and emplacement of ejecta by surface flow, J. Geophys. Res., 82, 4055-4065.

Cintala, M. J., and P. J. Mouginis-Mark (1980), Martian fresh crater depth: More evidence for subsurface volatiles, Geophys. Res. Lett., 7, 329-332.

Cintala, M. J., J. W. Head, and T. A. Mutch (1976), Martian crater depth/ diameter relationships: Comparison with the Moon and Mercury, Proc Lunar Sci. Conf. 7th, 3575-3587.

De Hon, R. A. (1982), Martian volcanic materials: Preliminary thickness estimates in the Eastern Tharsis region, J. Geophys. Res., 87, 98219828

Draper, N., and H. Smith (1981), Applied Regression Analysis, 2nd ed., John Wiley, New York.

Garvin, J. B., and J. J. Frawley (1998), Geometric properties of Martian impact craters: Preliminary results from the Mars Orbiter Laser Altimeter, Geophys. Res. Lett., 25, 4405-4408.
Garvin, J. B., S. H. E. Sakimoto, J. J. Frawley, and C. Schnetzler (2000), North polar region craterforms on Mars: Geometric characteristics from the Mars Orbiter Laser Altimeter, Icarus, 144, 329-352.

Gault, D. E., and R. Greeley (1978), Exploratory experiments of impact craters formed in viscous targets: Analogs for Martian rampart craters, Icarus, 34, 486-495.

Greeley, R. (1987), Release of juvenile water on Mars: Estimated amounts and timing associated with volcanism, Science, 236, 1652-1654.

Horner, V. M., and R. Greeley (1987), Effects of elevation and ridged plains thickness on Martian crater ejecta morphology, Proc. Lunar Planet. Sci. Conf. 17th, Part 2, J. Geophys. Res., 92, suppl., E561-E569

Mouginis-Mark, P. J. (1979), Martian fluidized crater morphology: Variations with crater size, latitude, altitude and target material, J. Geophys. Res., 84, 8011-8022.

Mouginis-Mark, P. J., and J. N. Hayashi (1993), Shallow and deep fresh impact craters in Hesperia Planum, Mars, Earth Moon Planets, 61, 1-20.

Mouginis-Mark, P. J., J. M. Boyce, V. E. Hamilton, and F. S. Anderson (2003), A very young, large, impact crater on Mars, in Sixth International Conference on Mars [CD-ROM], abstract 3004, Lunar and Planet. Inst., Houston, Tex.

Neukum, G. (2004), The HRSC experiment in Mars orbit: First results, Lunar Planet. Sci., XXXV, abstract 1883.

Pike, R. J. (1980a), Control of crater morphology by gravity and target type: Mars, Earth, Moon, Proc. Lunar Planet. Sci. Conf. 11th, 21592189.

Pike, R. J. (1980b), Geometric interpretation of lunar craters, U.S. Geol. Surv. Prof. Pap., 1046-C.

Pike, R. J., and P. A. Davis (1984), Toward a topographic model of Martian craters from photoclinometry, Lunar Planet. Sci., XV, 645-646.

Press, W. H., S. A. Teukolsky, W. T. Vetterling, and B. P. Flannery (1992), Numerical Recipes in C, 2nd ed., 674 pp., Cambridge Univ. Press, New York.

Selby, S. M. (Ed.) (1973), CRC Standard Mathematical Tables, 21st ed., 714 pp., CRC Press, Boca Raton, Fla.

Sheskin, D. J. (1997), Handbook of Parametric and Nonparametric Statistical Procedures, 719 pp., CRC Press, Boca Raton, Fla.

Soderblom, L. A., C. D. Condit, R. A. West, B. M. Herman, and T. J. Kreidler (1974), Martian planetwide crater distributions: Implications for geologic history and surface processes, Icarus, 22, 239-263.

Stewart, S. T., J. D. O'Keefe, and T. J. Ahrens (2001), The relationship between rampart crater morphologies and the amount of subsurface ice, Proc. Lunar Planet. Sci. Conf. 32nd, abstract 2092.

Wood, C. A., J. W. Head, and M. J. Cintala (1978), Interior morphology of fresh Martian craters: The effects of target characteristics, Proc. Lunar Planet. Sci. Conf. 9th, 3691-3709.

Zuber, M. T., D. E. Smith, S. C. Solomon, D. O. Muhleman, J. W. Head, J. B. Garvin, J. B. Abshire, and J. L. Bufton (1992), The Mars Observer Laser Altimeter investigation, J. Geophys. Res., 97, 7781-7797.

S. M. Baloga, Proxemy Research, Laytonsville, MD 20882, USA

J. M. Boyce, H. Garbeil, and P. J. Mouginis-Mark, Hawaii Institute Geophysics and Planetology, University of Hawaii, 1680 East-West Road, Honolulu, HI 96822, USA. (pmm@higp.hawaii.edu)

C. S. E. Ui, Sociology Department, University of Washington, Seattle, WA 98195, USA. 